\title{
A zebrafish nanos-related gene is essential for the development of primordial germ cells
}

\author{
Marion Köprunner, ${ }^{1}$ Christine Thisse, ${ }^{2}$ Bernard Thisse, ${ }^{2}$ and Erez Raz ${ }^{1,3}$ \\ ${ }^{1}$ Max Planck Institute for Biophysical Chemistry, Germ Cell Development, 37077 Göttingen, Germany; ${ }^{2}$ Institut de Génétique \\ et Biologie Moléculaire et Cellulaire, CNRS/INSERM/ULP, BP 163, 67404 Illkirch cedex, CU de Strasbourg, France
}

\begin{abstract}
Asymmetrically distributed cytoplasmic determinants collectively termed germ plasm have been shown to play an essential role in the development of primordial germ cells (PGCs). Here, we report the identification of a nanos-like (nanos1) gene, which is expressed in the germ plasm and in the PGCs of the zebrafish. We find that several mechanisms act in concert to restrict the activity of Nanos1 to the germ cells including RNA localization and control over the stability and translatability of the RNA. Reducing the level of Nanos 1 in zebrafish embryos revealed an essential role for the protein in ensuring proper migration and survival of PGCs in this vertebrate model organism.
\end{abstract}

[Key Words: nanos; germ line; PGC; germ plasm; maternal RNA; cell migration; zebrafish]

Received July 19, 2001; revised version accepted September 3, 2001.

Two main strategies for germ cell specification have been described; in mouse (and by inference other mammals) and in urodele amphibians germ cells are thought to be specified through cellular interactions (Nieuwkoop 1969; Tam and Zhou 1996). Consistently, the formation of the founding population of PGCs in the mouse was shown to depend on the function of extracellular factors of the bone morphogenetic protein (BMP) family (Lawson et al. 1999; Tsang et al. 2001; Ying et al. 2001; Ying and Zhao 2001). In contrast to this strategy, in many other organisms from different phyla, inheritance of specific asymmetrically localized cytoplasmic components, known as germ plasm, appears to direct cells to the germ-line lineage (Houston and King 2000a; Knaut et al. 2000; Tsunekawa et al. 2000). Although the actual specification and formation of PGCs in mammals and in urodeles appear to be independent of germ plasm, material resembling nuage, germ plasm organelle, is found in germ cells of these organisms at later stages of their development (Eddy 1974; Ikenishi and Nieuwkoop 1978).

An important contribution for the understanding of the mechanisms of germ cell development in fish was the identification of the zebrafish vasa homolog (Yoon et al. 1997). In Drosophila, the vasa gene, which encodes an RNA helicase, is essential for the assembly of the germ plasm (Hay et al. 1988; Lasko and Ashburner 1988). In situ hybridization of four-cell stage zebrafish embryos

\footnotetext{
${ }^{3}$ Corresponding author.

E-MAIL eraz@gwdg.de; FAX 49-5512011504.

Article and publication are at http://www.genesdev.org/cgi/doi/10.1101/ $\operatorname{gad} .212401$.
}

showed specific localization of the vasa transcript in four stripes at the edges of the first two cleavage planes of the early zebrafish embryo (Yoon et al. 1997). At these early stages of development the vasa transcript was shown to reside within an electron dense, nuage-like structure characteristic of germ plasm in other organisms (Knaut et al. 2000). During gastrulation and somitogenesis stages, the vasa-positive PGCs migrate and form two bilateral rows of cells close to the position of the future gonad (Weidinger et al. 1999).

Another germ plasm component that was first identified in Drosophila is nanos RNA, which encodes an RNA binding zinc finger protein. nanos transcripts are enriched in the posterior of the early Drosophila embryo, in the region where the germ granules reside (Wang and Lehmann 1991). Furthermore, nanos RNA is translated exclusively in the posterior, thereby generating a protein gradient with high protein levels in the posterior (Gavis and Lehmann 1994). Interestingly, although PGCs formation does not require nanos activity, nanos-deficient PGCs develop abnormally. They fail to incorporate into the gonad, show premature activation of germ cell markers, exhibit abnormal morphology, and express mRNAs that are normally expressed in the soma (Kobayashi et al. 1996; Forbes and Lehmann 1998; Deshpande et al. 1999).

Two C. elegans homologs of nanos have been shown to be required for early development of PGCs in this organism (Subramaniam and Seydoux 1999). Both genes (nos-1 and nos-2) encode maternal RNAs that are preferentially maintained in the germ-line blastomeres. Similar to Drosophila, the function of the C. elegans nanos genes is not required for the formation of the PGCs or for 
Köprunner et al.

early specification of the germ cell fate. However, PGCs lacking the function of the two nanos genes are not maintained, and most of them eventually die. In addition to these redundant functions, nos-2, independent of nos1 , is required for efficient incorporation of the PGCs into the gonad.

The first vertebrate nanos-related gene, Xcat-2, was identified in Xenopus laevis (Mosquera et al. 1993). Xcat-2 RNA is transported along with the germ plasm to the vegetal cortex during oogenesis where it is associated with germinal granules in a process that depends on cisacting elements in the 3'UTR (Kloc et al. 2000). Although the mechanisms responsible for Xcat-2 localization have been thoroughly studied, the function of this conserved germ plasm component has not yet been determined. Another Xenopus germ plasm component is the RNA of the DAZ-like gene, Xdazl. The function of this gene is required for spermatogenesis in flies and humans (Reijo et al. 1995; Eberhart et al. 1996), oogenesis in the nematode (Karashima et al. 2000), and gametogenesis in both male and female mice (Ruggiu et al. 1997). Specific depletion of maternal Xdazl mRNA results in PGC migration defects, and ultimately, loss of PGCs at the tadpole stage (Houston and King 2000b).

To understand the molecular mechanisms governing PGC development in zebrafish we have isolated genes that are expressed in these cells during embryonic development. Here, we report the cloning of a germ plasm component, the zebrafish nanos 1 . We show that several levels of post-transcriptional control act in concert to restrict the function of this gene to the PGCs. Reducing the level of Nanos revealed an essential role for this molecule in early development of the PGCs in a vertebrate model organism.

\section{Results}

Identification of nanos1, a gene expressed in the PGCs of zebrafish

We have sought to identify new genes that are important for germ cell development in zebrafish by employing a whole-mount in situ hybridization screen approach. In the course of this screen, we isolated a nanos-related gene (nos1, for nomenclature, see Materials and Methods), which is expressed in the PGCs of zebrafish (Figs. 1, $2 \mathrm{a}-\mathrm{e})$. The zebrafish nos 1 mRNA is provided maternally (Fig. 2a,h). During the first two mitotic divisions, the mRNA is enriched in the distal end of the cleavage furrows of the early embryo, while nonlocalized mRNA can be still detected by whole-mount in situ hybridization until late blastula stages (Fig. 2a,b). This expression pattern is similar to that described for the zebrafish vasa mRNA, a germ-cell specific marker. At these early stages of development, vasa transcrips are concentrated in an electron-dense structure, the putative zebrafish germ
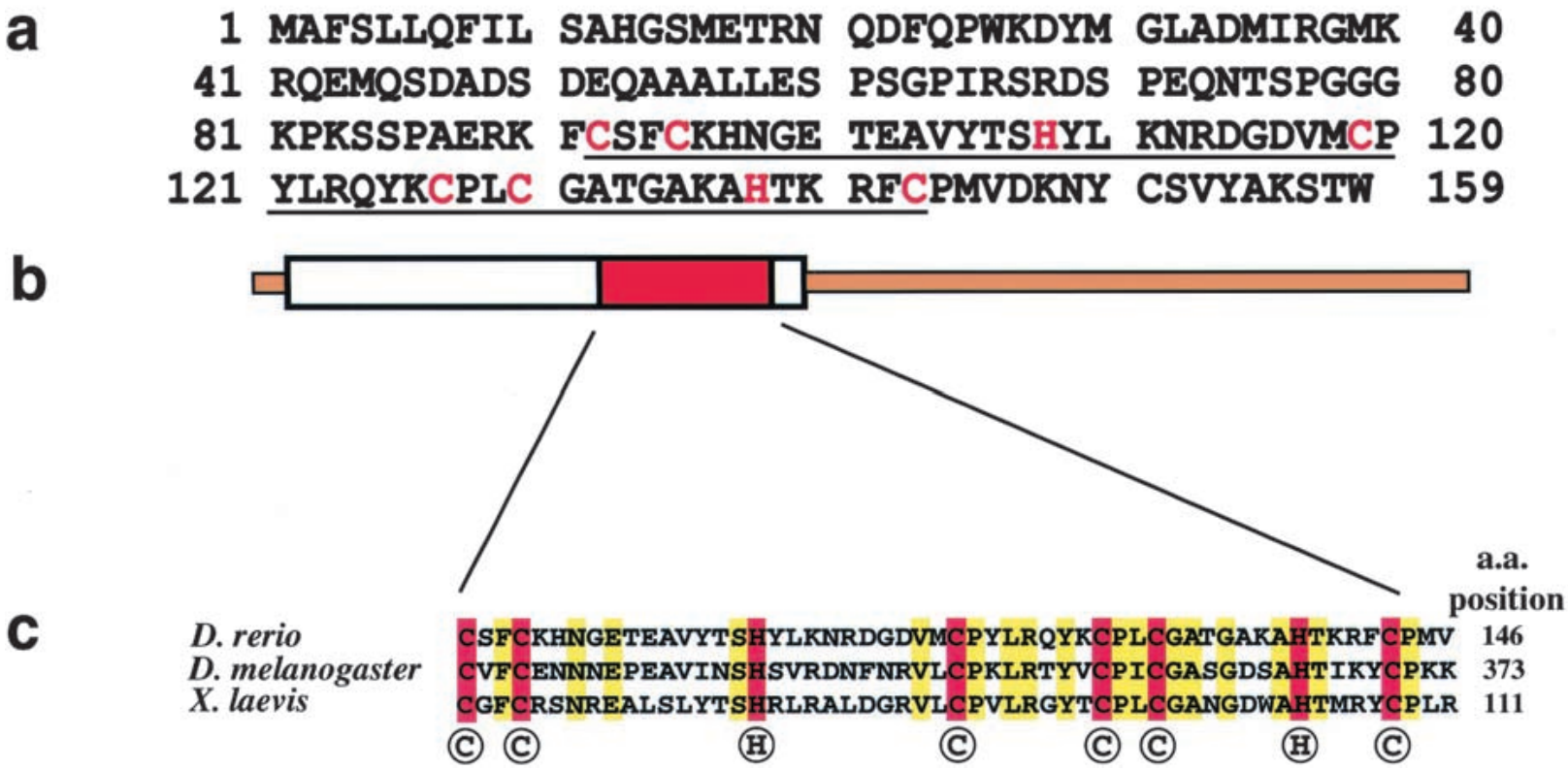

Figure 1. The zebrafish nanos 1 (nos1) gene encodes a nanos-like zinc finger protein. (a) The predicted amino acid sequence of Nanos1 (Nos1) protein. The characteristic C-terminal CCHC CCHC zinc finger domain with its conserved residues (red) is underlined. This is the only region that shows high homology to nanos genes of other organisms. $(b)$ Schematic representation of the cDNA of nos 1 . The cDNA contains 29 base pairs (bp) of 5'UTR (orange). The 480 bp-long open reading frame is followed by 637 bp of 3'UTR (orange). The red box within the coding region depicts the position of the zinc finger domain. (c) Comparison of the amino acid sequence of the zinc finger domain among Nanos-like proteins from Danio rerio (Nos1), Drosophila melanogaster (Nanos), and Xenopus laevis (Xcat-2). The amino acid residues comprising the conserved CCHC zinc finger are labeled in red, and residues that are identical in the three proteins are labeled in yellow. The total length of the Nanos1 protein is more similar to that of the Xcat-2 protein than to the Nanos proteins of Drosophila and C. elegans. 

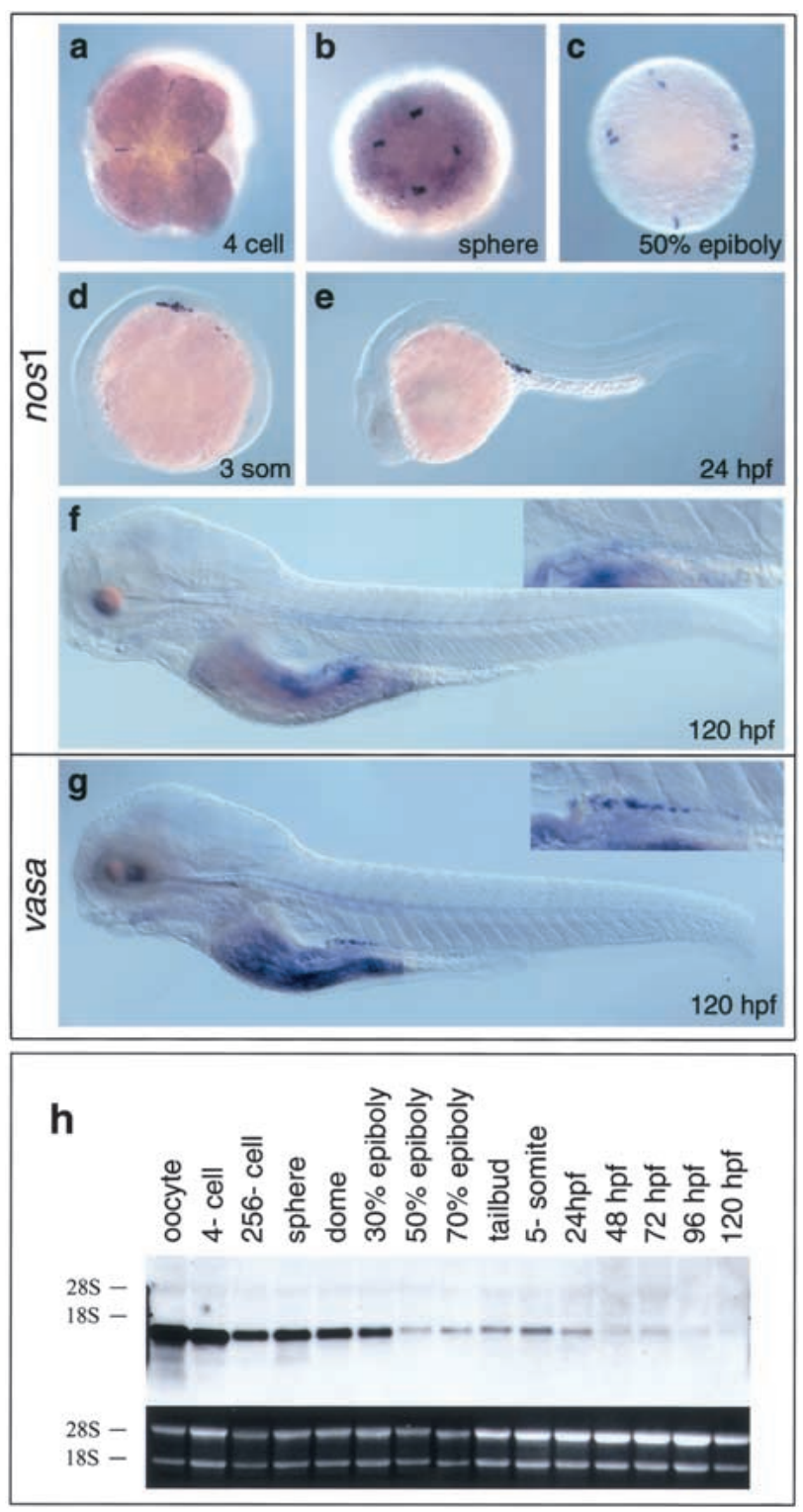

Figure 2. Spatial and temporal distribution of nos 1 RNA and Nos1 protein. $(a-f)$ Expression of nos 1 mRNA during the first 5 $\mathrm{d}$ of zebrafish development. nos $1 \mathrm{mRNA}$ is undetectable by the fifth day of development. ( $g$ ) Expression of vasa mRNA in a 5 -day-old embryo. Inserts in $f$ and $g$ show a magnification of the region where the PGCs are normally found. (h) Developmental Northern blot of nos1. nos 1 RNA is present in oocytes and in embryos before MBT (the 4- and 256-cell stages). The level of the transcript declines and it is barely detectable by the fifth day of development.

plasm (Yoon et al. 1997; Knaut et al. 2000). During blastula stages, nos 1 mRNA is incorporated into the PGCs, which characteristically migrate to form two clusters during the first day of development (Fig. 2c-e; Weidinger et al. 1999). Unlike vasa, which continues to be strongly expressed throughout germ-cell development, nos 1 mRNA is undetectable by in situ hybridization by the fifth day of embryonic development (Fig. $2 \mathrm{f}, \mathrm{g})$.
Restriction of Nanos1 activity to the PGC by posttranscriptional control mechanisms

In the one-cell stage zebrafish embryo, nuage-like structures are evenly distributed in small particles adjacent to the actin cortex of the cell (Knaut et al. 2000). This configuration rapidly changes during the first two cell divisions. In the two- and four-cell embryos the nuage-like particles are increased in size, and are seen in close association with microtubules and mitochondria just below the distal part of the first two cleavage furrows (Knaut et al. 2000). Thus, within a period of 30 to $40 \mathrm{~min}$, nuage structures that have been evenly distributed around the cortex of the large one-cell stage zebrafish embryo, accumulate in four aggregates. Several findings suggest that during the formation of these aggregates not all of the germ plasm material is transferred to the four main clusters. Although reduced in number, at the fourcell stage nonlocalized small nuage-like particles can still be observed along the actin cortex (Knaut et al. 2000). Indeed, vasa RNA, which labels the germ plasm, could be clearly detected up to the 64-cell stage outside of the four main germ plasm clusters (Yoon et al. 1997), and at later stages up to the sphere stage (late blastula, 4 hours postfertilization [hpf]; E. Raz and U. Wolke, unpubl.). Similarly, as mentioned above, nanos 1 mRNA can be detected outside of the four clusters up to late blastula stages (Fig. 2a,b). Thus, although enrichment of germ plasm is observed in the positions where the PGCs will form, a significant amount of nonlocalized germ plasm particles can be found in cells that undergo somatic differentiation at later stages. These findings, coupled with the fact that up to the 16-cell stage cells of the zebrafish embryo are interconnected (Kimmel et al. 1995), call for control mechanisms that would restrict the function of germ plasm components, RNA and proteins, to the future PGCs, allowing proper development of the germ line on one hand and an uninterrupted somatic differentiation on the other.

The spatial and temporal distribution of nos-1 mRNA during early embryogensis indicates that, indeed, posttranscriptional control mechanisms exist that facilitate the specific expression of the gene in the PGCs. Northern blot analysis shows that the maternally provided mRNA degrades rapidly before gastrulation (up to the $50 \%$ epiboly stage in Fig. $2 \mathrm{~h}$ ) and the level of the mRNA continues to decline to a very low level by the fifth day of development (Fig. 2h). The rapidly degrading mRNA is likely to represent transcripts that were not incorporated into the PGCs, because clearance of somatic nos 1 mRNA during these stages can be observed by wholemount in situ hybridization analysis (Fig. 2a-c). To study the regulation of nos 1 mRNA distribution more directly, we injected either full-length nos 1 mRNA tagged at its 5' end with green fluorescent protein (GFP) sequences (GFP-FLnos 1), or GFP mRNA fused to the 3'UTR of the nos1 gene (GFP-nos1-3'UTR). We then determined the spatial distribution of the fusion-RNA molecules by in situ hybridization using GFP as an RNA probe at different stages of development. The distribution pattern of 
the injected RNA derived from both fusion constructs was strikingly different from that of control RNA where GFP was fused to the 3'UTR of the Xenopus globin gene (GFP-globin). Specifically, whereas the control RNA showed slow uniform degradation in all cell types (Fig. $3 \mathrm{~m}-\mathrm{p})$, nos 1 3'UTR-containing RNAs exhibited rapid degradation in somatic tissues, while being specifically stabilized in the PGCs (Fig. 3e-h). Therefore, we conclude that the $3^{\prime} \mathrm{UTR}$ of nos 1 is sufficient for directing fast degradation of the transcript in the soma, as well as for specific protection of the RNA from degradation in the germ cells. We suggest that this mechanism acts on the maternal nos 1 mRNA to eliminate the ubiquitous distribution of the transcripts during early embryogenesis, thus leading to specific expression of nos 1 in the PGCs.

The significance of nos 1 3'UTR for Nos1 protein expression was determined by following the distribution of GFP in embryos injected with GFP-nos 1-3'UTR mRNA. We found that the $3^{\prime} \mathrm{UTR}$ of nos 1 is sufficient for directing specific expression of the protein in the PGCs despite the early ubiquitous distribution of the mRNA. In blastula stages, GFP was expressed uniformly, reflecting the spatial distribution of the injected RNA, but at a very low level (Fig. 3a,e). At this early stage the GFP signal derived from the similarly distributed GFP-globin

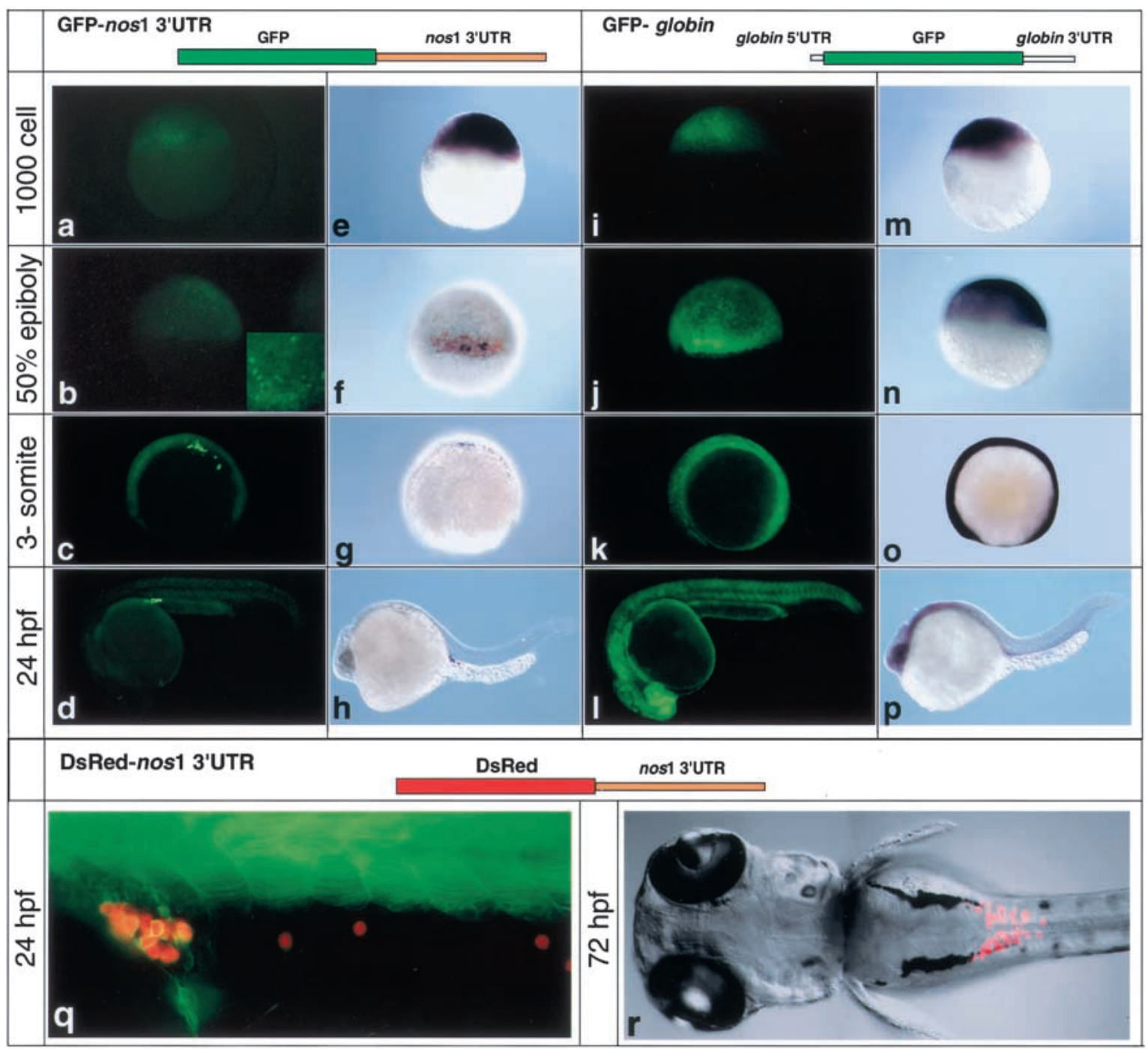

Figure 3. nos 1 RNA distribution and protein expression are regulated by its $3^{\prime} \mathrm{UTR}$. $(a-h)$ Embryos injected with 40 pg of GFP-nos 1 3'UTR RNA, or with 40 pg of GFP-globin RNA $(i-p) \cdot(a-d, i-1)$ Fluorescent pictures of live embryos injected with the constructs described above. The earliest time point when the germ cells can be distinguished from somatic cells is during early gastrulation stages (insert in $b) .(e-h, m-p)$ Whole-mount in situ hybridization of embryos injected with the constructs described above using GFP as a probe. $(e-h)$ Specific degradation in the soma and stabilization in the PGCs is observed for RNAs containing the nos 1 3'UTR. $(q)$ A 24-hour-old embryo injected with 100 pg of DsRed-nos1 3'UTR RNA at the one-cell stage showing red fluorescent in the PGC cluster with trailing posterior cells found along the yolk extension. Cell membranes were labeled by coinjecting 10 pg of EGFP-F-globin RNA. (r) Specific expression of DsRed in the PGCs of a 3-day-old embryo (dorsal view) injected with DsRed-nos1 3'UTR RNA at the one-cell stage. 
mRNA is significantly higher (Fig. 3 cf. a,e and i,m, where 3 a required a five times longer exposure time than 3i). A likely explanation for this phenomenon is that nos 1 mRNA is poorly translated in somatic cells. Differential GFP expression levels that distinguish between zebrafish germ cells and somatic cells can first be seen during early gastrulation stages (Fig. 3b). Specific GFP expression in the PGCs could be observed for several days after injection (for 24 hpf, see Fig. 3d). The control mechanisms that are responsible for concentrating Nanos protein in the PGCs appear to be conserved between Drosophila and zebrafish, because in Drosophila as well, nonlocalized RNA is translationally repressed and undergoes degradation (Gavis and Lehmann 1994; Bashirullah et al. 1999). Taken together, the tight control over the distribution of nos 1 mRNA through differential RNA stability in somatic versus germ-line cells and the control over the translation of nos 1 mRNA, results in PGC-specific Nos1 expression despite the early widespread RNA distribution in the early zebrafish embryo.

The ability to direct expression of specific genes to the PGCs in zebrafish using the nos 1 3'UTR has important practical implications for the study of vertebrate PGC. By using this procedure, one can now disrupt the normal function of specific genes in the PGCs (e.g., by gene overexpression or expression of dominant negative forms) to study their role in these cells. To show that proteins other than GFP can indeed be expressed in the PGCs using this method, we fused the cDNA encoding DsRed2 to the 3'UTR of nos 1 . Injection of this RNA led to specific expression of the protein in the PGCs, labeling them in red for more than $5 \mathrm{~d}$ (for $24 \mathrm{hpf}$ and 3-day-old embryos, see Fig. 3q,r). Labeling the PGCs with GFP allows the migration to be followed at high resolution in the physiological context in live embryos to obtain information regarding the dynamics of this process. Finally, sorting GFP-labeled PGCs will constitute an important step towards efficient culturing of these cells for the purpose of in vitro studies of PGCs as well as genetic manipulations such as transgenesis (Ma et al. 2001) and mutagenesis.

\section{Nanos1 is essential for proper migration and survival of PGCs in zebrafish}

As a first step in studying the function of the Nos1 protein we sought to determine its subcellular localization. In C. elegans, where the localization of a number of germ plasm components has been thoroughly studied, it appears these molecules are enriched in perinuclear granules that are, in turn, found in association with nuclear pores (Pitt et al. 2000). Similarly, in the zebrafish, Vasa protein is found in perinuclear granules in 1-day-old embryos (Knaut et al. 2000). The subcellular localization of Nos1 was determined by injecting mRNA containing the GFP open reading frame (ORF) fused in frame to the ORF of nos-1 (GFP-FLnos1) and following the distribution of GFP within the cell. Although the fusion protein is found throughout the cytoplasm, it is highly enriched in perinuclear granules that also contain the Vasa protein, suggesting nuclear-related functions for Nos1 (Fig. 4a).

To investigate the function of the Nos1 protein in zebrafish we inhibited its translation in early embryos using morpholino-modified antisense oligonucleotides (morpholinos) (Nasevicius and Ekker 2000). Remarkably, as will be described in detail below, embryos injected with nos 1 morpholinos exhibited severe defects in PGC development leading to a reduced number of cells and to migration defects (Fig. 4b). The specificity of the antisense oligonucleotides was confirmed by several experiments. First, morpholinos directed against the region of the translation start site of nos 1 efficiently inhibited the translation of synthetic nos 1 RNA, but did not affect the translation of synthetic RNAs lacking sequences complementary to it (data not shown). Second, the severity of the phenotype correlated with the concentration of the injected antisense oligonucleotides (Fig. 4c). Third, the morpholino-induced PGC phenotype was fully reversed by coinjection of modified nos 1 mRNA that could not be recognized by the antisense oligonucleotides (FLnos1mut) (Fig. 4e). Thus, uniformly supplied nos 1 RNA that is regulated by its 3'UTR is capable of restoring Nos 1 function in the germ cells. In contrast to the severe PGC phenotype induced by the morpholinos, under conditions where a PGC phenotype was observed, the development of the soma remained largely unaffected as determined by marker gene expression and morphological criteria (e.g., see Fig. 4b,f).

To elucidate the basis for the PGC phenotype in the nos 1 morpholino-injected embryos, we followed the position and the number of the PGCs during development using vasa or nos 1 probes in whole-mount in situ hybridization. Even when a very high amount of nos 1 morpholinos was injected $(2.4 \mathrm{ng})$, no change in PGC numbers at pregastrulation stages was observed ( $5 \mathrm{~h}$ of development, Fig. 4d). Therefore, we conclude that actual formation and specification of PGCs in zebrafish does not require zygotically translated Nos 1 protein. Remarkably, although reducing Nos1 level did not affect PGC formation, it did deleteriously affected their migration; in morpholino-injected embryos the PGCs failed to migrate normally, reaching ectopic positions such as within the forming somites and in the head region (Fig. 4f). The migration defects are apparent at the first migration step that presumably requires directed active PGC migration, as during gastrulation the PGCs fail to align along the border between the trunk and head mesoderm and along the lateral border of the mesoderm (step III; Weidinger et al. 1999). Interestingly, although the PGCs do not reach intermediate migration targets, the cells appear to be able to migrate in a nondirectional manner, and exhibit the morphological characteristics of migratory cells. In nos 1 morpholino-injected embryos the number of PGCs found in correct positions did not change significantly between 3 -somite (11 hpf) and 24 hpf stages, whereas the number of PGCs found in ectopic positions was dramatically reduced (Fig. 4f). In this context it is noteworthy to mention that wild-type PGCs are able to survive in ectopic positions while maintaining 


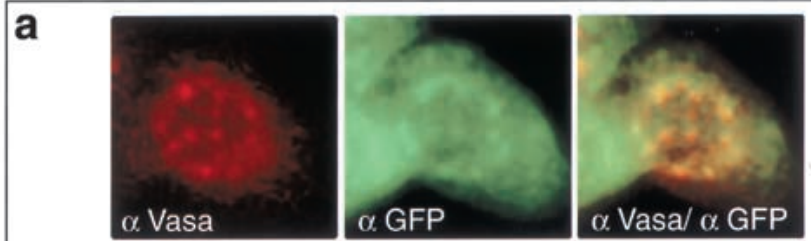

GFP-FLnos 1
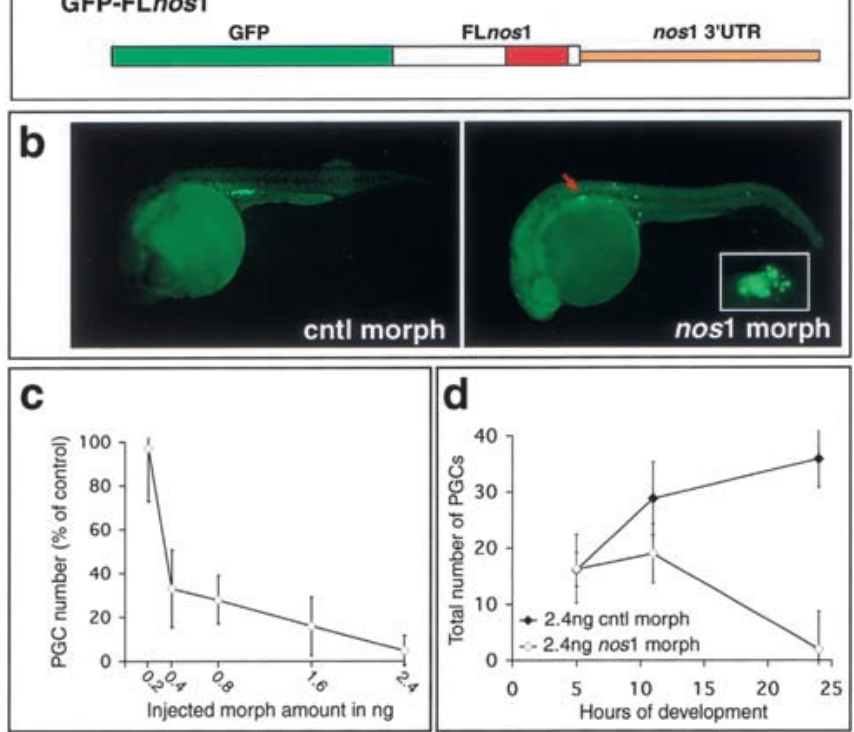
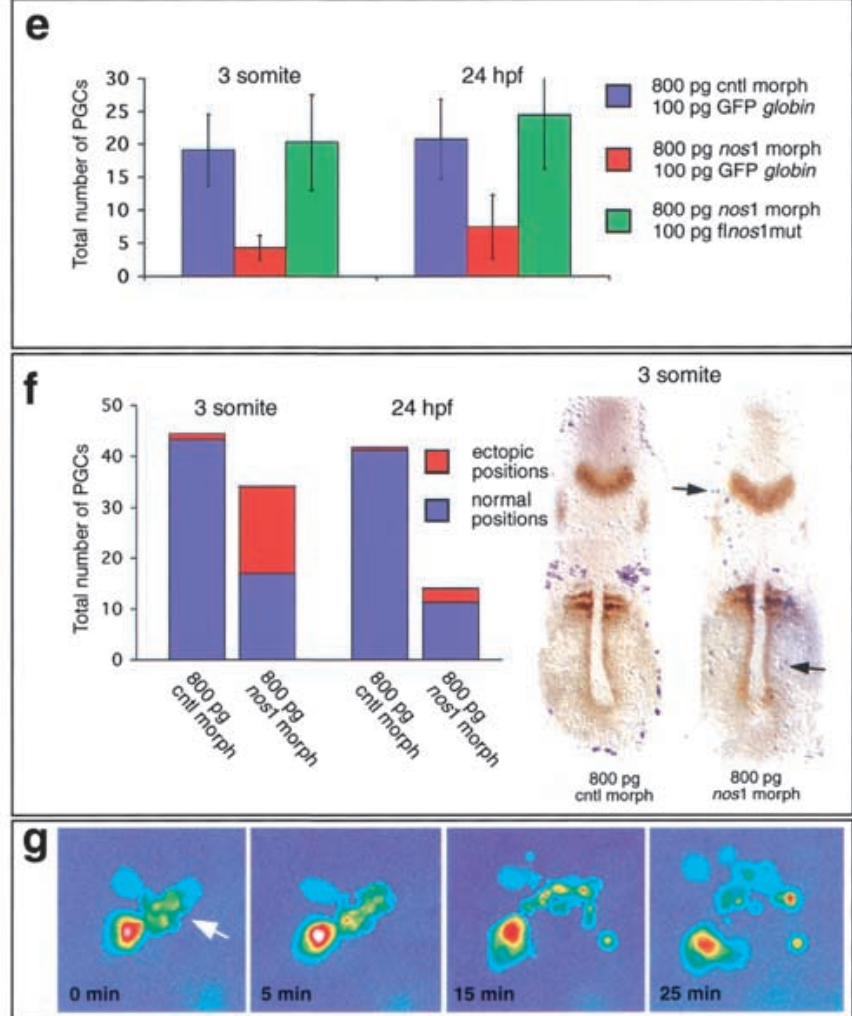

Figure 4. The function of Nos1 in PGC development in zebrafish. (a) The Nos1 protein is localized to perinuclear granules. Embryos injected with mRNA of GFP fused in frame to nos1 (200 pg of GFP-FLnos1) show localization of Nos1 to Vasa-containing granules (Knaut et al. 2000), while some of the protein appears to be distributed in the cytoplasm. (b) Defects in PGC migration and reduction in PGC number in nos 1 morpholino-injected embryos. Embryos were injected with 60 pg of GFP-nos 1 3'UTR and 800 pg of nos 1 morpholinos (right panel) or with $800 \mathrm{pg}$ of control morpholinos (left panel) and were photographed at the end of the first day of development. The insert in the right panel shows a magnification of a single dying ectopic PGC. The red arrow indicates the position of the cell within the embryo. (c) Loss of PGCs as a result of increased amounts of injected morpholinos. PGCs were visualized by whole-mount in situ hybridization using vasa as a probe at the 24-hpf stage. The proportion of PGC number in the nos 1 morpholinoinjected embryos relative to that of control morpholino-injected embryos is given for each experimental point (9-20 embryos were analyzed per point). (d) Loss of PGCs in nos 1 morpholino-injected embryos during development. The number of PGCs was determined using vasa as an in situ hybridization probe at the indicated developmental stages. Even at the highest concentration used, the initial number of PGCs was not affected by the injected nos 1 morpholinos (for each point 10-20 embryos were analyzed). (e) nos 1 morpholinoinjected embryos can be rescued by coinjection of nos 1 mRNA that cannot be bound by the nos 1 morpholino (FLnos 1 mut). $(f)$ PGCs of nos 1 morpholino-injected embryos are found in ectopic positions and die. The total number of PGCs decreases from the 3-somite stage to the 24-hpf stage, and most of the cell death can be attributed to cells found in ectopic locations (9-16 embryos were analyzed for each experimental point). Despite the severe PGC phenotype, whole-mount in situ hybridization of 3-somite stage embryos injected with morpholinos and stained with myoD, papC, pax2.1, pax 8 (all in brown) and vasa (blue) show that somatic development is largely unaffected (right panel, ectopic cells are labeled with arrows). (g) A dying PGC in nos 1 morpholino-injected embryos show the characteristic morphology of apoptotic cells. Embryos were injected with nos 1 morpholinos as in $b$ (right panel) and their PGCs were followed in live embryos at the end of gastrulation (1-somite stage). One of the two PGCs remains alive and exhibits normal morphology, while the other cell (arrow) undergoes cell death. Pseudocolors represent GFP intensity from blue (low) to green, yellow, red, and white (high).

typical PGC characteristics for several days (Weidinger et al. 1999). Taken together, these observations suggest that ectopic PGCs failed to migrate properly and then die due to the more severe reduction in the level of Nos1 protein relative to the cells that migrated properly. All of the ectopic cells exhibited morphological abnormalities. The dying GFP-labeled ectopic PGCs exhibited reduction in fluorescent intensity, membrane blebbing, nuclear fragmentation, followed by fast formation of apoptotic bodies (Fig. 4g). Morphological changes of this kind are characteristic of cells undergoing programmed cell death (Rich et al. 1999).

\section{Discussion}

With mammals and urodele amphibians being an exception, vertebrates exhibit asymmetric localization of cytoplasmic determinants - the germ plasm - that was suggested to play a role in specification and development of their PGCs (Houston and King 2000a; Knaut et al. 2000; 
Tsunekawa et al. 2000). In contrast to a large number of genes known to be important for germ plasm formation and PGC development in invertebrates, only few such genes have been characterized so far in vertebrates (Saffman and Lasko 1999; Wylie 1999; Houston and King 2000a). In Drosophila, many germ plasm components have been isolated employing genetic screens for maternal effect mutations. However in zebrafish, maternal effect screens are relatively difficult to perform and therefore are not likely to identify a large proportion of these genes (Pelegri and Schulte-Merker 1999). The identification of PGC-specific genes on the basis of sequence similarity to genes of other organisms is in some cases complicated by the low conservation of the primary sequence and by definition, such a strategy would not lead to the isolation of novel genes. In this study we utilized an alternative approach and present our results describing the first gene of several isolated as based on their specific expression in the PGCs of zebrafish (C. Thisse, B. Thisse, and E. Raz, unpubl.). Isolation of genes using this methodology coupled with the ability to efficiently block their translation in the early embryo is a powerful avenue for the identification and functional analysis of molecules important for germ plasm function and PGC development in vertebrates.

As mentioned above, germ plasm has not been identified in mammals and the pertaining evidence suggests that inherited asymmetrically-localized cytoplasmic factors have no role in PGC specification (e.g., Tam and Zhou 1996; Zernicka-Goetz 1998; Lawson et al. 1999; Tsang et al. 2001; Ying and Zhao 2001; Ying et al. 2001). Nevertheless, it appears that germ plasm-specific molecules identified in either invertebrates or vertebrates (e.g., vasa and Xdazl) do play a role at later stages of germ-cell development in mammals, as they are required for gametogenesis (Ruggiu et al. 1997; Tanaka et al. 2000). Thus, the identification and functional analysis of genes involved in early PGC development in zebrafish are relevant for the understanding of germ-line development in mammals, as well.

In this report, the function of a vertebrate nanos gene, a putative germ plasm component, is demonstrated for the first time. In zebrafish, nanos is required for the migration of PGCs and for the maintenance of their fate. These functions are strikingly similar to those of nanos during PGC development in invertebrates (Kobayashi et al. 1996; Forbes and Lehmann 1998; Deshpande et al. 1999; Subramaniam and Seydoux 1999). However, it is noteworthy that it is formally possible that a maternallyprovided protein whose level is not affected by the antisense oligonucleotides masks an earlier role of Nanos in zebrafish PGC development. This matter could be investigated by using anti-Nanos antibodies and by generating mutations in the nanos locus.

In order to restrict Nos1 to the PGCs of zebrafish, several control mechanisms are operating in concert at the level of asymmetric RNA localization, differential RNA stability and translation. Interestingly, in invertebrates the spatial distribution of Nanos is similarly controlled (e.g. Gavis and Lehmann 1994 ; Bashirullah et al. 1999).
Since other PGC-specific RNA molecules in zebrafish exhibit similar spatial distribution (Yoon et al. 1997; C. Thisse, B. Thisse, and E. Raz, unpubl.), it is conceivable that the mechanisms described here for nanos serve to restrict the function of other proteins to the PGCs as well. Taken together, our findings show that key steps in PGC development in invertebrates and in vertebrates, that is, PGC migration and maintenance of the PGC fate, require the function of related molecules, which are regulated by similar mechanisms.

\section{Materials and methods \\ Isolation of the nos-1 and nomenclature of nanos-related genes in zebrafish}

The nos 1 cDNA (GenBank accession no. AY052376) was isolated in a large-scale screen for genes that are differentially expressed during early zebrafish development (C. Thisse and B. Thisse, unpubl.). As judged by $3^{\prime}$ and $5^{\prime}$ RACE analysis, the original clone included the full-length cDNA. Another nanosrelated gene (nos2) had already been identified as an expressed sequence tag (EST, GenBank accession no. ai585000). Expression of this mRNA is first detected in the forming somites and in specific domains in the nervous system. No expression of this gene was detected in the germ cells during the first $2 \mathrm{~d}$ of development.

\section{Whole-mount in situ hybridization and antibody staining}

RNA whole-mount in situ hybridization using the nanos1, vasa, and other probes for somatic derivatives was performed as previously described (Weidinger et al. 1999). Immunhistochemistry was performed as described by Knaut et al. (2000). The monoclonal mouse anti-GFP (B-2) antibody (Santa Cruz Biotechnology) was used at a 1:500 dilution and rabbit anti-vasa antibody (Knaut et al. 2000) was diluted 1:5000 before use. Secondary antibodies, goat anti-mouse Alexa Flour 488 and goat anti-rabbit Alexa Flour 546 (Molecular Probes) were used at a 1:200 dilution.

\section{Plasmid constructs}

FLnos1mut This construct was used for rescuing nos 1 morpholino-injected embryos. The region that is recognized by the morpholino antisense oligonucleotide was mutated by introducing nine nucleotide exchanges that do not alter the encoded protein sequence. nos 1 cDNA served as the template for PCR amplification using the following forward primer: ATATAA

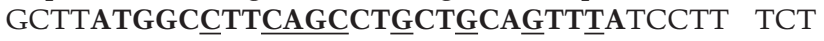
GCTCATGGAT (the region normally recognized by the morpholino oligonucleotide is in bold, and underlined residues designate positions of nucleotide exchanges relative to the wildtype sequence). The amplification product was cloned into pSP64T (Krieg and Melton 1984), from which the Xenopus globin UTRs were removed.

GFP-FLnos1 The construct includes mmGFP5 (Siemering et al. 1996) cloned into the 5'UTR of nos 1 in frame with the full nos1 ORF followed by the full nos1 3'UTR. The fused cDNA was cloned into pSP64T lacking the Xenopus globin UTRs.

GFP-nos1-3'UTR The construct includes the mmGFP5 ORF fused to the 3'UTR of nos 1 . The 3'UTR of nos 1 was cloned 3' to 
mmGFP5 in pSP64T from which the Xenopus globin UTRs were removed.

DsRed-nos1-3'UTR This construct was cloned similarly to GFP-nos1-3'UTR using the DsRed2 (Clonetech) protein as a dominant marker.

GFP-globin mmGFP5 was cloned in between the globin 5'UTR and globin 3'UTR of pSP64T.

EGFP-F-globin The cDNA of farnesylated EGFP (Clonetech), which is subcellularly localized to the plasma membrane, was cloned in between the globin 5'UTR and globin 3'UTR of the RN3 RNA expression vector (Lemaire et al. 1995).

\section{Microinjection into zebrafish embryos}

mRNA for injection was prepared using the mMessage Machine kit (Ambion), was diluted in $10 \mathrm{mM}$ HEPES (pH 7.6) and microinjected into one- to four-cell stage $\mathrm{AB}$ zebrafish embryos at the quantities indicated in the text and the figure legends. nos 1 morpholino (nos $1 \mathrm{morph}$ ) (TGAATTGGAGAAGAGAAAAAG CCAT) and control morpholino (cntl) (CCTCTTACCTCAG TTACAATTTATA) (Gene Tools) were injected in $10 \mathrm{mM}$ HEPES ( $\mathrm{pH}$ 7.6) at the quantities indicated in the text.

\section{Acknowledgments}

We thank V. Heyer and T. Steffan for technical assistance, and M. Reichman, G. Weidinger, U. Wolke, K. Artinger, and E. Gavis for critical reading of the manuscript. We thank H. Knaut and C. Nüsslein-Volhard for anti-Vasa antibodies. E.R is supported by grants from the DFG and from the Volkswagen-Stiftung. B.T. and C.T. are supported by funds from the Institut National de la Santé et de la Recherche Médicale, the Centre National de la Recherche Scientifique, the Hôpital Universitaire de Strasbourg, the Association pour la Recherche sur le Cancer, and the Ligue Nationale Contre le Cancer.

The publication costs of this article were defrayed in part by payment of page charges. This article must therefore be hereby marked "advertisement" in accordance with 18 USC section 1734 solely to indicate this fact.

\section{References}

Bashirullah, A., Halsell, S., Cooperstock, R., Kloc, M., Karaiskakis, A., Fisher, W., Fu, W., Hamilton, J., Etkin, L., and Lipshitz, H. 1999. Joint action of two RNA degradation pathways controls the timing of maternal transcript elimination at themidblastula transition in Drosophila melanogaster. EMBO J. 18: 2610-2620.

Deshpande, G., Calhoun, G., Yanowitz, J., and Schedl, P. 1999. Novel functions of nanos in downregulating mitosis and transcription during the development of the Drosophila germline. Cell 99: 271-281.

Eberhart, C.G., Maines, J.Z., and Wasserman, S.A. 1996. Meiotic cell cycle requirement for a fly homologue of human deleted in Azoospermia. Nature 381: 783-785.

Eddy, E. 1974. Fine structural observations on the form and distribution of nuage in germ cells of the rat. Anat. Rec. 178: $731-758$.

Forbes, A. and Lehmann, R. 1998. Nanos and Pumilio have critical roles in the development and function of Drosophila germline stem cells. Development 125: 679-690.

Gavis, E.R. and Lehmann, R. 1994. Translational regulation of nanos by RNA localization. Nature 369: 315-318.

Hay, B., Jan, L., and Jan, Y. 1988. A protein component of Drosophila polar granules is encoded by vasa and has extensive sequence similarity to ATP-dependent helicases. Cell 55: $577-587$.

Houston, D. and King, M. 2000a. Germ plasm and molecular determinants of germ cell fate. Curr. Top. Dev. Biol. 50: 155181.

2000b. A critical role for Xdazl, a germ plasm-localized RNA, in the differentiation of primordial germ cells in Xenopus. Development 127: 447-456.

Ikenishi, K. and Nieuwkoop, P. 1978. Location and ultrastructure of primordial germ cells (PGCs) in Ambystoma mexicanum. Dev. Growth Differ. 20: 1-9.

Karashima, T., Sugimoto, A., and Yamamoto, M. 2000. Caenorhabditis elegans homologue of the human azoospermia factor DAZ is required for oogenesis but not for spermatogenesis. Development 127: 1069-1079.

Kimmel, C.B., Ballard, W.W., Kimmel, S.R., Ullmann, B., and Schilling, T.F. 1995. Stages of embryonic development of the zebrafish. Dev. Dynam. 203: 253-310.

Kloc, M., Bilinski, S., Pui-Yee Chan, A., and Etkin, L.D. 2000. The targeting of Xcat2 mRNA to the germinal granules depends on a cis-acting germinal granule localization element within the 3'UTR. Dev. Biol. 217: 221-229.

Knaut, H., Pelegri, F., Bohmann, K., Schwarz, H., and NussleinVolhard, C. 2000. Zebrafish vasa RNA but not its protein is a component of the germ plasm and segregates asymmetrically before germline specification. J. Cell Biol. 149: 875888.

Kobayashi, S., Yamada, M., Asaoka, M., and Kitamura, T. 1996. Essential role of the posterior morphogen nanos for germline development in Drosophila. Nature 380: 708-711.

Krieg, P.A. and Melton, D.A. 1984. Functional messenger RNAs are produced by SP6 in vitro transcription of cloned cDNAs. Nucleic Acids Res. 12: 7057-7070.

Lasko, P. and Ashburner, M. 1988. The product of the Drosophila gene vasa is very similar to eukaryotic initiation factor-4A. Nature 335: 611-617.

Lawson, K.A., Dunn, N.R., Roelen, B.A., Zeinstra, L.M., Davis, A.M., Wright, C.V., Korving, J.P., and Hogan, B.L. 1999. $\mathrm{Bmp} 4$ is required for the generation of primordial germ cells in the mouse embryo. Genes \& Dev. 13: 424-436.

Lemaire, P., Garrett, N., and Gurdon, J.B. 1995. Expression cloning of siamois, a Xenopus homeobox gene expressed in dorsal-vegetal cells of blastulae and able to induce a complete secondary axis. Cell 81: 85-94.

Ma, C., Fan, L., Ganassin, R., Bols, N., and Collodi, P. 2001. Production of zebrafish germ-line chimeras from embryo cell cultures. Proc. Natl. Acad. Sci. 98: 2461-2466.

Mosquera, L., Forristall, C., Zhou, Y., and King, M.L. 1993. A mRNA localized to the vegetal cortex of Xenopus oocytes encodes a protein with a nanos-like zinc finger domain. $D e$ velopment 117: 377-386.

Nasevicius, A. and Ekker, S.C. 2000. Effective targeted gene "knockdown" in zebrafish. Nat. Genet. 26: 216-220.

Nieuwkoop, P.D. 1969. The formation of mesoderm in Urodelean amphibians. I. Induction by the endoderm. Wilhelm Roux Arch. EntwMech. Org. 162: 341-373.

Pelegri, F. and Schulte-Merker, S. 1999. A gynogenesis-based screen for maternal-effect genes in the zebrafish, Danio rerio. Methods Cell Biol. 60: 1-20.

Pitt, J., Schisa, J., and Priess, J. 2000. P granules in the germ cells of Caenorhabditis elegans adults are associated with clusters of nuclear pores and contain RNA. Dev. Biol. 219: 315333. 
Reijo, R., Lee, T.Y., Salo, P., Alagappan, R., Brown, L.G., Rosenberg, M., Rozen, S., Jaffe, T., Straus, D., Hovatta, O., et al. 1995. Diverse spermatogenic defects in humans caused by Y chromosome deletions encompassing a novel RNA-binding protein gene. Nat. Genet. 10: 383-393.

Rich, T., Watson, C., and Wyllie, A. 1999. Apoptosis: The germs of death. Nat. Cell Biol. 1: E69-E71.

Ruggiu, M., Speed, R., Taggart, M., McKay, S.J., Kilanowski, F., Saunders, P., Dorin, J., and Cooke, H.J. 1997. The mouse Dazla gene encodes a cytoplasmic protein essential for gametogenesis. Nature 389: 73-77.

Saffman, E.E. and Lasko, P. 1999. Germline development in vertebrates and invertebrates. Cell. Mol. Life Sci. 55: 11411163.

Siemering, K., Golbik, R., Sever, R., and Haseloff, J. 1996. Mutations that suppress the thermosensitivity of green fluorescent protein. Curr. Biol. 6: 1653-1663.

Subramaniam, K. and Seydoux, G. 1999. nos-1 and nos-2, two genes related to Drosophila nanos, regulate primordial germ cell development and survival in Caenorhabditis elegans. Development 126: 4861-4871.

Tam, P. and Zhou, S. 1996. The allocation of epiblast cells to ectodermal and germ-line lineages is influenced by the position of the cells in the gastrulating mouse embryo. Dev. Biol. 178: 124-132.

Tanaka, S.S., Toyooka, Y., Akasu, R., Katoh-Fukui, Y., Nakahara, Y., Suzuki, R., Yokoyama, M., and T. Noce. 2000. The mouse homolog of Drosophila Vasa is required for the development of male germ cells. Genes \& Dev. 14: 841-853.

Tsang, T.E., Khoo, P.L., Jamieson, R.V., Zhou, S.X., Ang, S.L. Behringer, R., and Tam, P.P. 2001. The allocation and differentiation of mouse primordial germ cells. Int. J. Dev. Biol. 45: 549-555.

Tsunekawa, N., Naito, M., Sakai, Y., Nishida, T., and Noce, T. 2000. Isolation of chicken vasa homolog gene and tracing the origin of primordial germ cells. Development 127: 27412750.

Wang, C. and Lehmann, R. 1991. Nanos is the localized posterior determinant in Drosophila. Cell 66: 637-647.

Weidinger, G., Wolke, U., Koprunner, M., Klinger, M., and Raz, E. 1999. Identification of tissues and patterning events required for distinct steps in early migration of zebrafish primordial germ cells. Development 126: 5295-5307.

Wylie, C. 1999. Germ cells. Cell 96: 165-174.

Ying, Y. and Zhao, G.Q. 2001. Cooperation of endoderm-derived BMP2 and extraembryonic ectoderm-derived BMP4 in primordial germ cell generation in the mouse. Dev. Biol. 232: 484-492.

Ying, Y., Qi, X., and Zhao, G.Q. 2001. Induction of primordial germ cells from murine epiblasts by synergistic action of BMP4 and BMP8B signaling pathways. Proc. Natl. Acad. Sci. 98: 7858-7862.

Yoon, C., Kawakami, K., and Hopkins, N. 1997. Zebrafish vasa homologue RNA is localized to the cleavage planes of 2- and 4-cell-stage embryos and is expressed in the primordial germ cells. Development 124: 3157-3165.

Zernicka-Goetz, M. 1998. Fertile offspring derived from mammalian eggs lacking either animal or vegetal poles. Development 125: 4803-4808. 


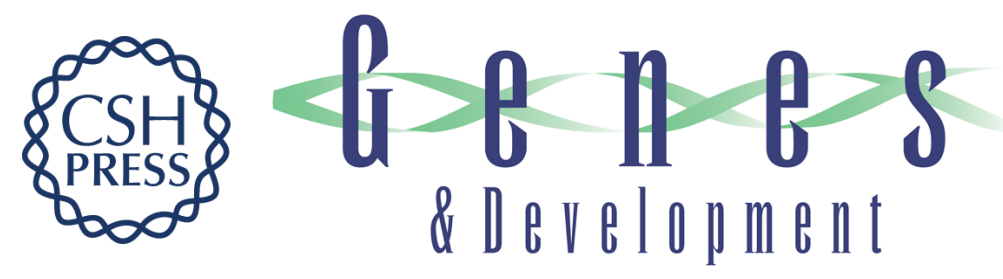

\section{A zebrafish nanos-related gene is essential for the development of primordial germ cells}

Marion Köprunner, Christine Thisse, Bernard Thisse, et al.

Genes Dev. 2001, 15:

Access the most recent version at doi:10.1101/gad.212401

References This article cites 41 articles, 15 of which can be accessed free at: http://genesdev.cshlp.org/content/15/21/2877.full.html\#ref-list-1

License

Email Alerting

Receive free email alerts when new articles cite this article - sign up in the box at the top Service right corner of the article or click here.

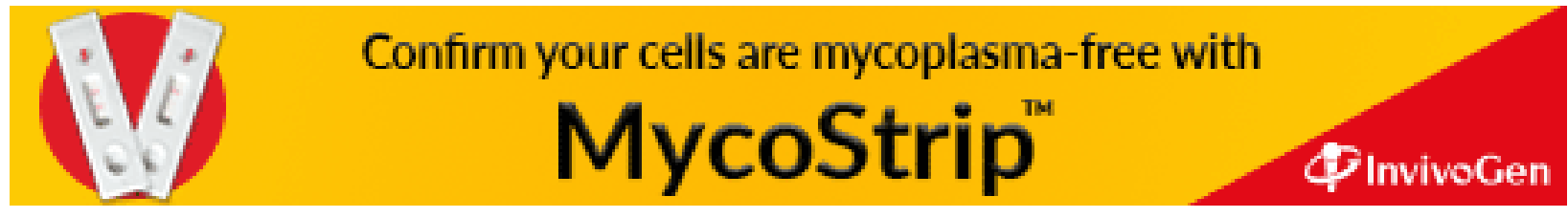

